\title{
Adopting genetics: motivations and outcomes of personal genomic testing in adult adoptees
}

\author{
Natalie M. Baptista, BSc (Hons) ${ }^{1,2}$, Kurt D. Christensen, PhD ${ }^{1}$, \\ Deanna Alexis Carere, ScD, CGC1,3, Simon A. Broadley, MD, PhD², \\ J. Scott Roberts, PhD ${ }^{4}$ and Robert C. Green, MD, MPH ${ }^{1,5,6,7}$; for the PGen Study Group
}

Purpose: American adult adoptees may possess limited information about their biological families and turn to direct-to-consumer personal genomic testing (PGT) for genealogical and medical information. We investigated the motivations and outcomes of adoptees undergoing PGT using data from the Impact of Personal Genomics (PGen) Study.

Methods: The PGen Study surveyed new 23andMe and Pathway Genomics customers before and 6 months after receiving PGT results. Exploratory analyses compared adoptees' and nonadoptees' PGT attitudes, expectations, and experiences. We evaluated the association of adoption status with motivations for testing and postdisclosure actions using logistic regression models.

Results: Of 1,607 participants, 80 (5\%) were adopted. As compared with nonadoptees, adoptees were more likely to cite limited knowledge of family health history $(\mathrm{OR}=10.1 ; 95 \% \mathrm{CI}=5.7-19.5)$ and the opportunity to learn genetic disease risks (OR $=2.7 ; 95 \%$ $\mathrm{CI}=1.6-4.8)$ as strong motivations for PGT. Of 922 participants who completed 6-month follow-up, there was no significant association between adoption status and PGT-motivated health-care utilization or health-behavior change.

Conclusion: PGT allows adoptees to gain otherwise inaccessible information about their genetic disease risks and ancestry, helping them to fill the void of an incomplete family health history.

Genet Med advance online publication 28 January 2016

Key Words: adult adoptee; adoption; direct-to-consumer genetic testing; family history; personal genomic testing

\section{INTRODUCTION}

Family health history is a cornerstone of modern medicine, ${ }^{1,2}$ but for some patients, this information is unavailable. Direct-to-consumer personal genomic testing (PGT) is one way for these individuals to obtain personalized information regarding disease risks, inherited traits, pharmacogenomics, and ancestry. ${ }^{3-5}$ However, the provision of genetic information directly to customers who have limited family-history information underlies one of the primary criticisms of PGT: without a family history through which to contextualize PGT results, and without clinician interpretation, consumers may be falsely reassured by low-risk results or unnecessarily alarmed by results indicating an elevated risk of disease. ${ }^{6-8}$

Adopted individuals constitute one group whose health care may be affected by a lack of family-history information, ${ }^{9-11}$ but they can face ethical and practical challenges when attempting to obtain genetic-risk information. ${ }^{12}$ Anecdotal reports from PGT companies and in the media suggest that adoptees have used PGT from Family Tree DNA and 23andMe to find biological family members and to learn about their ancestry. ${ }^{4,13}$ Beyond this, little is known about PGT customers who were adopted, and there is no consensus on the appropriateness of disclosing genetic results to those with limited family-history information. Because adoptees may differ from the general population in their responses to PGT, there is a need for empirical study of how adoptees perceive and utilize PGT results. ${ }^{10}$

Using data from the Impact of Personal Genomics (PGen) Study ${ }^{14}$ a longitudinal study of PGT customers of $23 \mathrm{andM} \mathrm{e}^{15}$ and Pathway Genomics ${ }^{16}$ (Pathway), we conducted an exploratory analysis comparing adoptees and nonadoptees who used PGT. We compared cohorts with respect to baseline (pre-disclosure) demographics, psychosocial characteristics, family health-history knowledge, and motivations for seeking PGT. At 6 months after disclosure of PGT results to customers, we compared adoptees and nonadoptees with respect to PGT-motivated health-care utilization and PGT-motivated health-behavior change. Finally, we analyzed adoptees' perceptions of the value of PGT.

\section{MATERIALS AND METHODS}

\section{Participants}

The PGen Study is a collaboration between academic researchers and industry scientists from 23andMe and Pathway Genomics. Details of the study's design and methodology, ${ }^{14,17}$

${ }^{1}$ Division of Genetics, Department of Medicine, Brigham and Women's Hospital, Boston, Massachusetts, USA; ${ }^{2}$ School of Medicine, Gold Coast Campus, Griffith University, Queensland, Australia; ${ }^{3}$ Program in Genetic Epidemiology and Statistical Genetics, Harvard T.H. Chan School of Public Health, Boston, Massachusetts, USA; ${ }^{4}$ Department of Health Behavior and Health Education, University of Michigan School of Public Health, Ann Arbor, Michigan, USA; ${ }^{5}$ Harvard Medical School, Boston, Massachusetts, USA; ${ }^{6}$ Partners Personalized Medicine, Boston, Massachusetts, USA; ${ }^{7}$ Broad Institute, Boston, Massachusetts, USA. Correspondence: Robert C. Green (rcgreen@genetics.med.harvard.edu) 
along with other reports from the study, ${ }^{18-20}$ have previously been published. Briefly, new customers of 23andMe and Pathway were recruited between March and July 2012. They provided online consent to participate in the study, including consent to link survey responses with PGT results. Prior to receiving their PGT results, 1,648 participants completed a baseline survey. Participants were followed for 6 months after receiving their results. A diagram summarizing the PGen Study design, including the timing of measures and exclusions relevant to these analyses, is provided in the supplementary information (Supplementary Figure S1 online). The study protocol was approved by the Partners Human Research Committee.

\section{Personal and family history}

Adoptees and nonadoptees were identified using two items in the baseline survey. Participants were asked, "Are you adopted?" and responded "yes" or "no." They also reported whether "desire to learn more about my genetics because I am adopted" was a motivation to seek PGT. Participants who responded "yes" to the first item were classified as adoptees. Participants who responded "no" to the first item and "very important" or "somewhat important" to the second item were classified as having an unclear adoption status. Participants with an unclear adoption status who consented to additional contact were contacted by phone to confirm whether they were adopted; if their status remained unclear, these participants were omitted from further analyses.

Demographic information was collected via self-report. Self-reported health was measured on a 5-point scale derived from the 36-item Short Form Health Survey ("excellent" = 1 to "poor" = 5). ${ }^{21}$ Body mass index was calculated using participants' self-reported height and weight. Frequency of exercise was measured using a question adapted from the National Health Interview Survey, ${ }^{22}$ in response to which participants reported the number of days per week ( $0-7$ days) they performed vigorous exercise for at least 10 minutes. Fruit and vegetable consumption was measured by asking participants to report how many servings of each they consumed on a typical day $(0,1,2,3,4$, or $5+)$, with responses of " $5+$ " recoded as "5." Participants' anxiety and depression levels were assessed using the two-item Generalized Anxiety Disorder (GAD-2) scale $^{23}$ and the two-item Patient Health Questionnaire (PHQ-2) score ${ }^{24}$ respectively. Higher scores (range $=0-6$ ) indicated greater anxiety/depression. A positive emotions score (0-6) was calculated as the sum of responses to two Mental Health Inventory ${ }^{25}$ items rated on a 4-point scale. Higher scores indicated greater frequency of positive emotions.

Level of family health-history knowledge about first-, second-, and third-degree relatives was ascertained by asking participants whether any of their blood relatives had a history (yes or no) of 15 specific conditions.

\section{Genetic testing}

Exposure to genetic testing and genetics specialists was assessed by asking participants if they had ever met clinically with a genetic counselor or genetics specialist, undergone previous genetic testing other than newborn screening, or previously purchased PGT from a different company. A five-item genetics selfefficacy scale, ${ }^{18}$ with a Cronbach's $\alpha$ value of approximately 0.94 , was used to calculate a genetics self-efficacy score. Participants rated their agreement with each item using a 7-point Likert scale ("strongly disagree" = 1 to "strongly agree" =7), and the ratings were summed to give a score ranging from 5 to 35 .

Participants reported how important 11 motivations were to their decision to seek PGT using 3-point scales ("very important," "somewhat important," or "not at all important") and provided open-ended responses articulating why they sought PGT. On 3-point scales, participants rated seven factors in their decision to seek PGT ("considered a lot," "considered somewhat," or "did not consider") and their interest in learning about four types of PGT results ("very interested," "somewhat interested," or "not at all interested").

At 6 months after disclosure of PGT results, participants were asked with whom they had discussed their results. Participants could choose from family, friends, co-workers, medical professionals (primary-care provider, genetics specialist, or other), and social-networking contacts. Healthcare utilization was measured by creating a composite yes/ no variable whereby "yes" represented a participant who affirmed that their PGT results prompted any medical tests, examinations, or procedures or consultations with a medical professional. Health-behavior change was measured by creating a composite yes/no variable whereby "yes" represented a participant who affirmed that their PGT results prompted any change in diet, exercise, medications (prescription, nonprescription, or alternative), or use of vitamins/supplements. Weekly vigorous exercise and daily fruit and vegetable consumption were measured again at 6 months after disclosure using the same questions asked at baseline.

At 6 months after disclosure, participants also provided open-ended responses explaining why they thought the PGT experience was valuable or not. A 5-point scale ("not at all" = 1 to "extremely" = 5) was used to assess how valuable participants found their results and how satisfied they were with their decision to seek PGT. Decision regret was assessed using a validated five-item scale (range $=0-100),{ }^{26}$ with higher scores indicating greater regret about the decision to undergo PGT.

\section{Data analysis}

We excluded participants from all analyses if they viewed their PGT results prior to completing the baseline survey, had an unclear adoption status, or were missing required baseline data. We excluded from follow-up analyses participants who were missing required 6-month survey data for variables used to determine health-care utilization or health-behavior changes.

Descriptive statistics were computed to summarize the characteristics of the study sample. Data were stratified by adoption status. Categorical variables were compared using chi-squared tests. Continuous variables were compared using Welch's $t$-tests, which allow for unequal variances within cohorts. Statistical significance was set at $P<0.05$. 


\section{ORIGINAL RESEARCH ARTICLE}

For logistic or linear regression analyses of baseline variables, bivariate or multivariate regression was undertaken as appropriate. To facilitate analysis, motivations were dichotomized to "very important" versus "somewhat"/"not important," decision-making factors were dichotomized to "considered a lot" versus "considered somewhat"/"not considered," and informational interests were dichotomized to "very interested" versus "somewhat"/"not interested." Each of these outcomes was regressed on adoption status using bivariate and multivariate logistic regression.

For logistic or linear regression analyses of 6-month outcomes, bivariate or multivariate regression was also undertaken as appropriate. Discussion of PGT results, health-care utilization, and health-behavior changes were analyzed as dichotomous variables, with regression on adoption status in bivariate and multivariate logistic regression models. Changes from baseline in vigorous physical exercise levels and daily fruit and vegetable consumption were compared by adoption status using Welch's $t$-tests after confirming normal distributions. Correlation tests were also conducted to determine whether reported PGT-motivated changes in exercise and diet were associated with changes in frequency of vigorous exercise and daily fruit and vegetable consumption from baseline to 6-month follow-up. Satisfaction and value responses were dichotomized to "extremely"/"very" versus "somewhat"/"a little"/ "not at all," and differences by adoption status were analyzed using chi-squared tests. Owing to skewed distributions, decision regret was analyzed as a dichotomous variable of scores of 0 and scores greater than 0 . Differences in decision regret by adoption status were analyzed using bivariate and multivariate logistic regression.

Emergent themes were identified from adoptees' free-form responses describing why they underwent PGT and whether they found it to be valuable. Themes were identified by generating word-frequency lists, followed by an analysis of key words in context performed by the first author. ${ }^{27}$

Multivariate analyses were adjusted for biological children, PGT company, prior PGT, and demographics that differed by cohort (age, gender, education, race, ethnicity). Statistical significance was set at $P<0.05$. Data analyses were performed using R software (version 3.2.0; R Foundation for Statistical Computing, Vienna, Austria).

\section{Code availability}

The computer code used for statistical analyses is available from the corresponding author upon request.

\section{Baseline survey sample}

\section{RESULTS}

The baseline analyses of 1,607 participants included 80 adoptees and 1,527 nonadoptees. Participants who completed the baseline questionnaire but had an unclear adoption status ( $n=24)$ or missing data for descriptive and motivational questions ( $n=17$, all nonadoptees) were excluded from the analyses (Supplementary Figure S1 online).
Compared with nonadoptees, adoptees appeared to be, on average, younger and less highly educated, with fewer biological children, higher body mass index, and lower daily fruit intake, although the difference in number of biological children was not significant when controlled for age $(P=0.209)$. Adoptees were also more likely to be customers of 23andMe (Table 1). Adoptees reported fewer positive emotions than nonadoptees in the 2 weeks prior to completing the baseline survey, but anxiety and depression scores did not differ.

Adoptees reported fewer conditions affecting blood relatives than nonadoptees (mean $=3.3$ among adoptees versus 6.8 among nonadoptees, $P<0.001$ ) (Supplementary Figure S2 online). Conditions for which adoptees most frequently reported having an affected blood relative were substance abuse (40\%), cancer (37\%), and heart conditions (34\%). By contrast, nonadoptees most frequently reported having an affected blood relative with cancer (79\%), heart conditions (73\%), and high cholesterol (70\%). Nonadoptees were more likely to report an affected blood relative for 9 of 15 queried conditions (Supplementary Table S1 online, all $P \leq 0.001)$. Data were missing for 18 adoptees and 32 nonadoptees (Supplementary Table S2 online).

\section{Motivations and considerations when purchasing PGT}

Adoptees and nonadoptees were both strongly motivated to purchase PGT because they were curious about their genetic makeup (Table 2). Compared with nonadoptees, adoptees were more motivated by their limited knowledge of their family health history and desire to learn their personal disease risk. Half of adoptees and nonadoptees factored actionability of PGT results into their decision to purchase PGT, and adoptees were less likely to consider genetic privacy ( $23 \%$ of adoptees versus $41 \%$ of nonadoptees, $\mathrm{OR}=0.4,95 \%$ confidence interval $(\mathrm{CI}): 0.2-0.7, P=0.001)$. Both groups were keenly interested in learning about ancestry and disease risk and less interested in learning about carrier status. Bivariate analyses suggested that adoptees were less interested in learning pharmacogenomic information than nonadoptees, although the differences were not significant in adjusted analyses.

Several themes emerged from analysis of adoptees' open-ended responses describing why they sought PGT (Table 3), but in all the themes they acknowledged their lack of personal or familial information, using language such as "no access to knowledge." They also indicated that they had turned to PGT to search for their missing information. Adoptees expressed a long-standing desire to learn about their ancestry: "I have always wanted to know more about my background" and "I have always felt a desire to know where I come from." Adoptees' lack of knowledge about their genetic makeup motivated their search for personal genetic-risk information; for example, one reported wanting to know "relative risk for diseases and adverse pharmacogenetic interactions." Adoptees wanted to learn familial risks for family planning purposes and to educate offspring, and some wanted to find and contact biological family members: "this service will be a long shot to connect with them." 
Table 1 Descriptive statistics of adopted and nonadopted PGen study participants at baseline

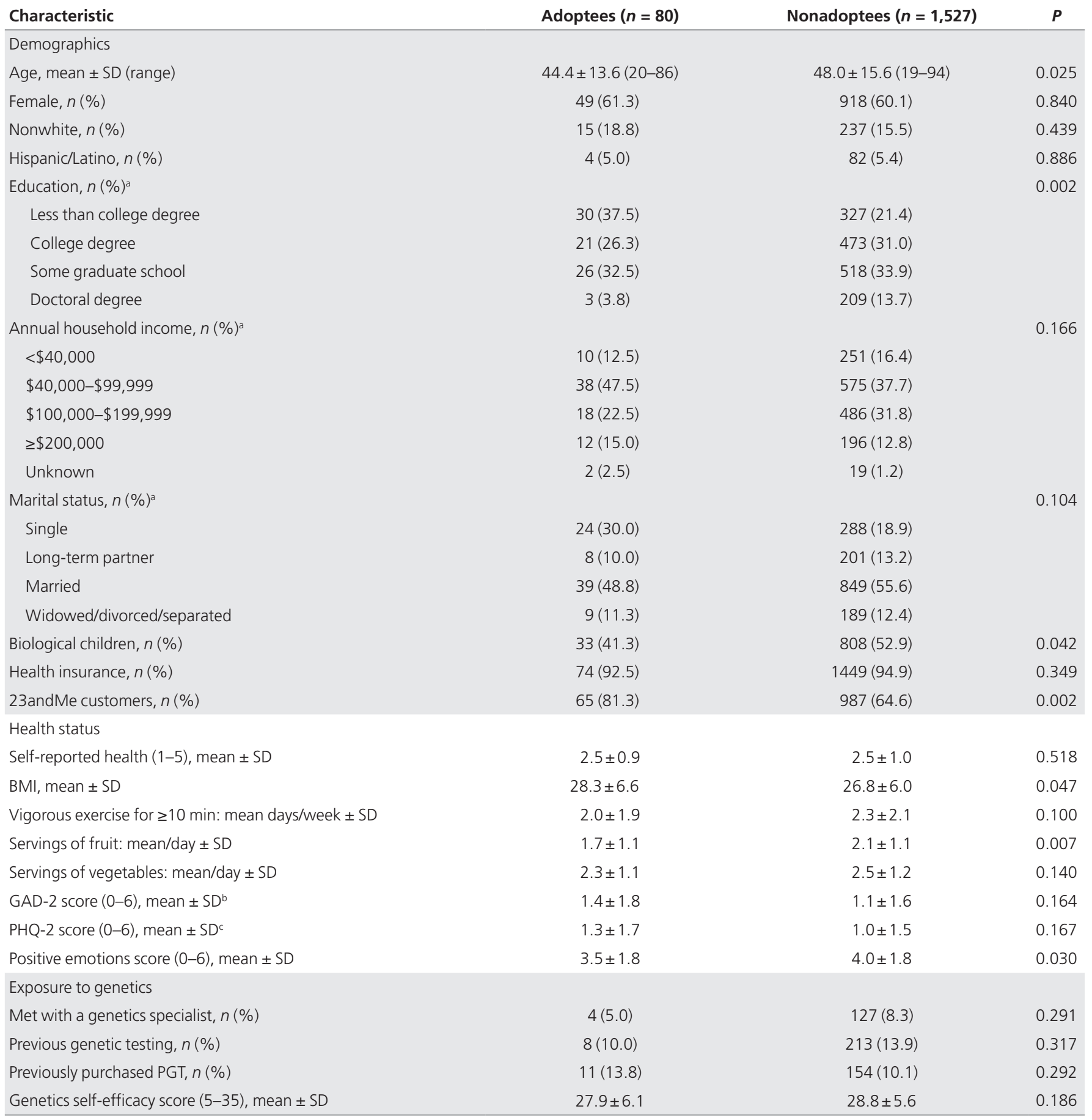

$\mathrm{BMI}$, body mass index; GAD, generalized anxiety disorder; PHQ, patient health questionnaire; PGT, personal genomic testing.

${ }^{a}$ Chi-squared tests were used to obtain global $P$ values for categorical variables. ${ }^{\circ}$ The GAD-2 was used to assess a participant's level of anxiety and higher scores indicated greater anxiety. 'The PHQ-2 was used to assess a participant's level of depression and higher scores indicated greater depression.

\section{The six-month follow-up survey sample}

Six-month follow-up survey data were analyzed for 51 adoptees and 871 nonadoptees $(n=922)$ after excluding participants who were missing required 6-month survey data for variables used to determine health-care utilization or healthbehavior changes ( $n=6$ adoptees and $n=97$ nonadoptees)
(Supplementary Figure S1 online). No evidence of differential attrition or exclusion by adoption status was observed, with $64 \%$ of adoptees and $57 \%$ of nonadoptees included in analyses $(P=0.237)$.

A comparison of the 922 participants who were included in 6-month analyses and the 685 participants who did not 
Table 2 Logistic regression analyses of motivations, decision-making factors, and informational interests when seeking PGT (by adoption status)

\begin{tabular}{|c|c|c|c|c|c|c|}
\hline \multirow[b]{2}{*}{ Baseline survey item } & \multirow{2}{*}{$\begin{array}{c}\begin{array}{c}\text { Adoptees } \\
(n=80)\end{array} \\
n(\%)\end{array}$} & \multirow{2}{*}{$\begin{array}{c}\text { Nonadoptees } \\
(n=1,527)\end{array}$} & \multicolumn{2}{|c|}{$\begin{array}{c}\text { Unadjusted } \\
\text { bivariate analysis }\end{array}$} & \multicolumn{2}{|c|}{$\begin{array}{l}\text { Adjusted }{ }^{\text {logistic }} \\
\text { regression analysis }\end{array}$} \\
\hline & & & OR $(95 \% \mathrm{Cl})$ & $P$ & OR $(95 \% \mathrm{Cl})$ & $P$ \\
\hline \multicolumn{7}{|l|}{ Motivations considered "very important" } \\
\hline Curiosity about my genetics & $67(84)$ & $1,188(78)$ & $1.5(0.8-2.8)$ & 0.212 & $1.3(0.7-2.5)$ & 0.793 \\
\hline Limited information about my family health history & $67(84)$ & $497(33)$ & $10.7(6.0-20.4)$ & $<0.001$ & $10.1(5.7-19.5)$ & $<0.001$ \\
\hline Interest in learning my personal risk of disease & $62(78)$ & $903(59)$ & $2.4(1.4-4.2)$ & 0.001 & $2.7(1.6-4.8)$ & $<0.001$ \\
\hline Personal interest in genetics in general & $38(48)$ & $828(54)$ & $0.8(0.5-1.2)$ & 0.241 & $0.7(0.4-1.1)$ & 0.153 \\
\hline Interest in learning my carrier status & $34(43)$ & $644(42)$ & $1.0(0.6-1.6)$ & 0.954 & $1.2(0.7-2.0)$ & 0.473 \\
\hline Desire to improve my health & $32(40)$ & $706(46)$ & $0.8(0.5-1.2)$ & 0.276 & $0.9(0.6-1.5)$ & 0.823 \\
\hline Desire to create a better plan for the future & $30(38)$ & $705(46)$ & $0.7(0.4-1.1)$ & 0.131 & $0.8(0.5-1.3)$ & 0.385 \\
\hline Interest in my personal pharmacogenomics & $28(35)$ & $600(39)$ & $0.8(0.5-1.3)$ & 0.444 & $1.1(0.6-1.8)$ & 0.830 \\
\hline Desire to learn about my genetics without going through a physician & $28(35)$ & $433(28)$ & $1.4(0.8-2.2)$ & 0.202 & $1.3(0.8-2.1)$ & 0.262 \\
\hline The service seemed fun and entertaining & $27(34)$ & $551(36)$ & $0.9(0.6-1.4)$ & 0.672 & $0.7(0.5-1.2)$ & 0.244 \\
\hline Other members of my family are PGT customers & $4(5)$ & $182(12)$ & $0.4(0.1-0.9)$ & 0.069 & $0.4(0.1-1.0)$ & 0.079 \\
\hline \multicolumn{7}{|l|}{ Decision-making factors "considered a lot" } \\
\hline Whether genetic information can inform health-related actions & $40(50)$ & $780(51)$ & $1.0(0.6-1.5)$ & 0.851 & $1.1(0.7-1.7)$ & 0.759 \\
\hline The convenience of being tested at home & $36(45)$ & $705(46)$ & $1.0(0.6-1.5)$ & 0.838 & $0.9(0.6-1.4)$ & 0.595 \\
\hline How well the results can predict my risk of disease & $27(34)$ & $459(30)$ & $1.2(0.7-1.9)$ & 0.484 & $1.2(0.7-1.9)$ & 0.507 \\
\hline Cost of services & $23(29)$ & $453(30)$ & $1.0(0.6-1.6)$ & 0.861 & $0.9(0.5-1.5)$ & 0.749 \\
\hline Privacy of my genetic information & $18(23)$ & $620(41)$ & $0.4(0.2-0.7)$ & 0.002 & $0.4(0.2-0.7)$ & 0.001 \\
\hline The education materials provided by the company & $14(18)$ & $358(23)$ & $0.7(0.4-1.2)$ & 0.222 & $0.7(0.3-1.2)$ & 0.160 \\
\hline The possibility of receiving unwanted information & $13(16)$ & $308(20)$ & $0.8(0.4-1.4)$ & 0.394 & $0.9(0.4-1.5)$ & 0.614 \\
\hline \multicolumn{7}{|l|}{ Information participants were "very interested" in learning } \\
\hline Ancestry & $66(83)$ & $1,116(73)$ & $1.7(1.0-3.3)$ & 0.066 & $1.3(0.7-2.5)$ & 0.405 \\
\hline Risk of disease or health condition & $63(79)$ & $1,095(72)$ & $1.5(0.9-2.6)$ & 0.174 & $1.6(0.9-2.8)$ & 0.116 \\
\hline Pharmacogenomics & $30(38)$ & $810(53)$ & $0.5(0.3-0.8)$ & 0.007 & $0.6(0.4-1.0)$ & 0.063 \\
\hline Carrier status & $25(31)$ & $471(31)$ & $1.0(0.6-1.6)$ & 0.939 & $1.0(0.6-1.6)$ & 0.849 \\
\hline
\end{tabular}

Each dichotomized baseline survey item was regressed on adoption status in bivariate and multivariate logistic regression models.

$\mathrm{Cl}$, confidence interval; OR, odds ratio; PGT, personal genomic testing.

aAll models adjusted for baseline age, gender, race, ethnicity, education, biological children, PGT company, and prior PGT.

complete the 6-month follow-up survey or were excluded from analyses is presented in Supplementary Table $\mathbf{S 3}$ online. Compared with participants omitted from analyses, participants whose 6-month data were analyzed tended to be younger (mean age $=46.6$ vs. $49.4, P<0.001$ ), were less likely to have biological children (49 vs. $57 \%, P=0.001$ ), were less likely to be 23 andMe customers ( 62 vs. $70 \%, P<0.001$ ), were more likely to have undergone previous genetic testing (16 vs. $11 \%$, $P=0.018$ ), and had higher mean genetics self-efficacy scores (29.1 vs. $28.2, P=0.003)$. Among adoptees, no statistically significant differences were observed between participants whose 6-month outcomes were analyzed compared with adoptees who were not analyzed (Supplementary Table S4 online). Among nonadoptees, characteristics of participants whose 6-month outcomes were analyzed and omitted followed patterns observed for the study sample overall (Supplementary Table S5 online).

\section{PGT-motivated actions and attitudes}

PGT results specifically motivated $41 \%$ of all participants to utilize a health-care service and $56 \%$ to change a health behavior (diet/exercise/medications/vitamins or supplements) within 6 months of receiving PGT results. Adoptees and nonadoptees reported similar levels of PGT-motivated health-care utilization and frequencies of health-behavior changes (Table 4). Analyses showed no difference between adoptees and nonadoptees with respect to mean change in vigorous exercise days per week $(-0.0$ vs. 0.1 , respectively, $P=0.511)$ from baseline to 6 months, although quantified changes were correlated with reported PGT-motivated exercise changes $(r=0.16, P<0.001)$. Analyses also showed no difference between groups with respect to mean change in daily fruit ( 0.2 vs. 0.1 , respectively, $P=0.309$ ) or vegetable ( 0.2 vs. 0.1 , respectively, $P=0.716$ ) servings, but, again, quantified changes in fruit $(r=0.08, P=0.012)$ and vegetable $(r=0.14, P<0.001)$ consumption were each correlated with 
Table 3 Illustrative quotes from adoptees explaining why they underwent PGT

\begin{tabular}{|c|c|}
\hline Theme & Quotes \\
\hline \multirow[t]{2}{*}{ Ancestry $(30)^{\mathrm{a}}$} & $\begin{array}{l}\text { "I was adopted a few days after birth and have no record of the ethnicity of my birth parents. I do not } \\
\text { undoubtably look any certain ethnicity, but my adoptive parents are both white so I've always stood out. I have } \\
\text { always wanted to know more about my background." (Woman, age 24) }\end{array}$ \\
\hline & $\begin{array}{l}\text { "The family that adopted me has always had a strong interest in their own personal heritage and genetics. } \\
\text { I have no information, and I have always felt the desire to know where I come from." (Woman, age 21) }\end{array}$ \\
\hline \multirow[t]{2}{*}{ Personal genetic risk (42) } & $\begin{array}{l}\text { "I am an adopted person with no access to knowledge of my genetic heritage or health background. It is } \\
\text { primarily because I want to know something about my own genetic makeup that I have done genetic testing." } \\
\text { (Man, age 58) }\end{array}$ \\
\hline & $\begin{array}{l}\text { "I am adopted and I have no information regarding family history of illness. I am primarily interested in my } \\
\text { relative risk for diseases and adverse pharmacogenetic interactions." (Man, age 23) }\end{array}$ \\
\hline \multirow[t]{2}{*}{ Familial risks (5) } & $\begin{array}{l}\text { "I am adopted and plan on having biological children with my wife. We wanted to get some indication of my } \\
\text { family medical history and genetic risk factors before we started the process." (Man, age 29) }\end{array}$ \\
\hline & $\begin{array}{l}\text { "I was adopted as a baby and can't get any information on my Bio family. I have been ill most of my life...I } \\
\text { wanted to be able to give my children some info so they are aware." (Woman, age 47) }\end{array}$ \\
\hline \multirow[t]{2}{*}{ Finding biological family members (9) } & $\begin{array}{l}\text { "I am adopted and have been denied information about my birth family although I have been given limited } \\
\text { information about their existence. This service will be a long shot to connect with them." (Man, age 42) }\end{array}$ \\
\hline & $\begin{array}{l}\text { "I discovered I was adopted three years ago. I have very limited information on my biological parents... the } \\
\text { relative finder portion of } 23 \text { andme.com is a great feature. I am hoping I have some relatives out there that have } \\
\text { used 23andMe's service." (Woman, age 34) }\end{array}$ \\
\hline
\end{tabular}

aNumbers in parentheses indicate the total number of quotes assigned to each theme. Seventy-five quotes were analyzed and quotes could be assigned to more than one theme. Five adoptees did not provide a free-form response.

Table 4 Logistic regression analyses of PGT results-motivated health-care utilization and health-behavior change (by adoption status)

\begin{tabular}{|c|c|c|c|c|c|c|}
\hline \multirow{2}{*}{$\begin{array}{l}\text { PGT results-motivated action reported at } \\
6 \text {-month follow-up }\end{array}$} & \multirow{2}{*}{$\begin{array}{c}\begin{array}{c}\text { Adoptees } \\
(n=51)\end{array} \\
n(\%)\end{array}$} & \multirow{2}{*}{$\begin{array}{c}\text { Nonadoptees } \\
(n=871)\end{array}$} & \multicolumn{2}{|c|}{$\begin{array}{l}\text { Unadjusted bivariate } \\
\text { analysis }\end{array}$} & \multicolumn{2}{|c|}{$\begin{array}{l}\text { Adjusted }{ }^{\mathrm{a}} \text { logistic } \\
\text { regression analysis }\end{array}$} \\
\hline & & & OR $(95 \% \mathrm{Cl})$ & $P$ & OR $(95 \% \mathrm{Cl})$ & $P$ \\
\hline Health-care utilization (consultations/tests) & $22(43)$ & $358(41)$ & $1.1(0.6-1.9)$ & 0.774 & $1.4(0.8-2.6)$ & 0.267 \\
\hline Health-behavior change (medication/exercise/diet) & $27(53)$ & $488(56)$ & $0.9(0.5-1.6)$ & 0.666 & $1.0(0.5-1.8)$ & 0.909 \\
\hline
\end{tabular}

Health-care utilization and health-behavior change were analyzed as dichotomous yes/no variables, with regression on adoption status in bivariate and multivariate logistic regression models.

$\mathrm{PGT}$, personal genomic testing; OR, odds ratio; $\mathrm{Cl}$, confidence interval.

aAll models adjusted for baseline age, gender, race, ethnicity, education, biological children, PGT company, and prior PGT.

Table 5 Illustrative quotes from adoptees describing why they found PGT to be valuable or not valuable

\section{Theme}

Gained otherwise inaccessible information (7)

Felt relieved after receiving genetic-risk results (2)

Desired more definitive risk information (4)

Disappointed by the lack of biological family members identified (2)

\section{Quotes}

"There is simply no other practical way to obtain this data. Even though its value in planning is limited and generally contains nothing that requires immediate action, it is still valuable." (Man, age 42)

"My medical history was a mystery for the first 20 years of my life. I wanted to see what I could glean from these tests to assist me in maintaining my health and improving it." (Woman, age 46)

"Gave a lot of information my adoptive family could not." (Woman, age 24)

"Put me at ease especially about cancer and diabetes tendencies." (Woman, age 54)

"Where I'm not a carrier for certain traits I felt relieved" (Woman, age 37)

"Many of the results were not clear-cut high or low." (Woman, age 60)

"Thought it may be more specific and less general." (Man, 56)

"Has not yet led me to any close matches. Most are 4th or 5th cousins and without any family history, I can't really tell anything." (Woman, age 44)

"I would have liked to have seen more close ancestry matches." (Man, age 50)

aNumbers in parentheses indicate the total number of quotes assigned to each theme. Twenty-six quotes were analyzed, and 25 adoptees did not provide a free-form response. 
reported PGT-motivated dietary changes. Adoptees appeared to be more likely than nonadoptees to discuss their results with co-workers (53\% of adoptees versus $34 \%$ of nonadoptees, $\mathrm{OR}=2.1,95 \%$ CI: $1.2-3.9, P=0.014)$ but no more likely to discuss their results with social-networking contacts, primary-care providers, family, or friends (all $P>0.05$ ).

The majority of adoptees and nonadoptees considered their PGT results valuable (69 vs. $62 \%$ ) and were satisfied with their decision to seek PGT (78 vs. $81 \%$ ). Furthermore, $71 \%$ of adoptees and $60 \%$ of nonadoptees scored the minimum for decision regret, and no difference in decision regret was observed between adoptees and nonadoptees in bivariate $(\mathrm{OR}=0.6,95 \%$ CI: $0.3-1.1, P=0.122)$ or adjusted analyses ( $\mathrm{OR}=0.6,95 \% \mathrm{CI}$ : $0.3-1.0, P=0.074)$.

An analysis of adoptees' open-ended responses describing why the PGT experience was valuable or not valuable is presented in Table 5. Adoptees considered PGT valuable because it provided a means of accessing information that was previously difficult for them to obtain. Some adoptees expected to receive more definitive genetic results, and others were disappointed that the PGT service had not revealed any close biological relatives.

\section{DISCUSSION}

Using data from the PGen Study, we have described the characteristics and motivations of adoptees undergoing personal genomic testing, and how adoptees use their genetic results. A strong desire for information is key to adoptees' beliefs and actions. The information obtained from PGT, including genetic risks, ancestry, and the identities of biological relatives, may be otherwise difficult to obtain for adoptees, whereas PGT provides a convenient and affordable method of access. Our results may help health-care professionals and policy makers to better understand the desires of adopted patients and how the provision of genetic information may affect their health. Although PGT results motivated many adoptees to change a health behavior or utilize a health-care service, they did so no more than nonadoptees did.

The adoptees in our sample knew less about their family health history than nonadoptees did, as might be expected. Adoptees experienced fewer positive emotions than nonadoptees in the 2 weeks prior to testing but were not more often depressed. A sociopsychological study by Sobol et al. ${ }^{28}$ found that adult adoptees who searched for birth parents, compared with nonsearchers, were more likely to believe that adoption made them feel different and incomplete. Adoptees may be more emotionally invested in discovering their genetic background than nonadoptees, and deciding to seek PGT may arouse more negative emotions for adoptees who are actively thinking about the family-history information they lack.

Both adoptees and nonadoptees were strongly motivated to purchase PGT because of curiosity about their genetics and an interest in learning their risk for specific diseases. Similar motivations of PGT customers have been reported. ${ }^{5,29}$ Adoptees were more strongly motivated to learn their genetic disease risks than nonadoptees, and this desire among adoptees may arise from their common experience of having an incomplete family history. A lack of family-history information may affect an adoptee's mental health, ${ }^{11}$ interfere with disease management and prevention, ${ }^{9,30}$ and result in stigmatization by medical professionals. ${ }^{9}$ Adoptees may recognize that they are at a health disadvantage because they lack certain family health-history information and are seeking genetic testing to improve their health outcomes.

Numerous blog posts and accounts in the news media have suggested that adoptees use PGT to obtain ancestry information and find biological relatives. ${ }^{4,13} \mathrm{~A}$ study by Crouch et al. ${ }^{30}$ that explored adoptive parents' attitudes toward whole-genome sequencing for their adopted children coincidently discovered that three participants had purchased PGT for their adopted children in an effort to address their children's questions about their background. Our results support the inference that obtaining ancestry information is a strong motivation for adult adoptees when purchasing PGT. A person's identity can be shaped by his or her ethnicity and ancestry, and lack of information in this area may be a concern for some adoptees. ${ }^{11}$ Adoptees were more likely to be 23 andMe customers than Pathway customers; 23andMe's PGT service may have appealed to adoptees because it offers ancestry information and a method of contacting biological relatives. We expected adoptees to be more interested in learning about ancestry than nonadoptees, but we found that interest in obtaining ancestry information was not more strongly associated with being adopted.

Some PGT companies integrated notions of autonomy and privacy into their marketing strategies, with advertising campaigns promoting direct, autonomous access to genetic information while bypassing the health-care system and avoiding inclusion of results in medical records. ${ }^{5,31}$ From this perspective, PGT could be perceived as a service that protects customer privacy. However, some PGT customers may inaccurately assume that confidentiality standards governing physician-patient interactions apply to PGT company-customer relationships, ${ }^{32}$ and customers may not be aware of their privacy vulnerabilities. Adoptees were less concerned about the privacy of their genetic information prior to testing than nonadoptees, and may have been more willing to share their results with people not closely related. Adoptees may be less concerned about privacy because they are actively trying to discover their genetic identity, and they may be frustrated about the secrecy surrounding their biological family's genetic information and therefore be advocating for easier access to adoption (and genetic) records.

Our evidence did not suggest that adoptees would be more likely than nonadoptees to base important health decisions on PGT results alone. PGT motivated adoptees and nonadoptees to utilize health-care services, such as consultations with medical professionals or medical tests, and to change a health behavior, such as changes in medication, diet, or exercise. Other studies have found that customers take similar health-related actions after receiving PGT results. ${ }^{33,34}$

Perhaps because PGT may be the only accessible source of genetic-risk and ancestry information for adoptees, the mere 
opportunity to receive such information was considered valuable. Adoptees who did not find PGT to be as valuable as expected were looking for more definitive information regarding their genetic risks. Su et al. ${ }^{5}$ suggested that some PGT users may overestimate its potential value. This may also be true of adoptees in our study, in cases in which a participant's unrealistic expectations prior to testing contributed to postdisclosure dissatisfaction.

PGT is unable to replace a comprehensive family-history assessment. Studies that have analyzed genetic-risk predictions from PGT have found inconsistencies between risk estimates and family medical histories. ${ }^{6,35}$ Aiyar et al. ${ }^{6}$ suggested that PGT results and family medical histories provide complementary rather than identical risk information, and Bloss et al. ${ }^{35}$ concluded that PGT results offer little added value beyond that which could be obtained through personal and family health-history information. It remains to be seen whether genetic-risk predictions from PGT can be useful in the absence of family-history information.

Strengths of our study include the longitudinal investigation of a large sample of PGT customers, the wide variety of survey questions, and lack of differential attrition. The PGen Study was not originally designed to compare the experiences of adoptees and nonadoptees who used PGT. As such, limitations of this study include unequal sample sizes of adoptees versus nonadoptees and limited power to detect differences between cohorts. Data were self-reported; and significance levels were not adjusted for multiple comparisons, increasing the risk of false-positive findings. Although all adoptees in our sample live in the United States, we do not know their place of birth, and, although it is likely, we cannot assume they were subject to restrictive adoption laws. Another limitation is that our assessment of family-history knowledge is not as comprehensive as a gold-standard family history in clinical practice. Items regarding PGT-motivated health-behavior change did not clearly indicate that such participant changes were positive, although context and associated data suggest that dietary and exercise changes were remedial. The PGen Study did not collect sufficient baseline data on prescription medication use and adherence to perform correlation analyses similar to those presented for PGT-motivated dietary and exercise changes. Data were collected regarding the frequency of physician visits at baseline, but we omitted this variable from the analyses because data were missing for $16(31 \%)$ individuals in the 6-month sample of adoptees. Finally, our findings are generalizable only to direct-to-consumer PGT customers similar to those enrolled in the PGen Study, who tended to be well educated, of high socioeconomic status, and white.

Our results emphasize the need for further study of the long-term health impact on adoptees who receive genetic information, particularly addressing any harms or unjustified health-related actions arising from the disclosure of genetic-risk results. Large, well-established, longitudinal adoption studies may be in the best position to study adoptees who use genetic testing services. These studies could analyze adoptees who have used, or who will receive, PGT or other genetic testing.

In conclusion, we conducted an exploratory analysis comparing adoptees and nonadoptees who used PGT using data from the longitudinal PGen Study. Adoptees used PGT to gain otherwise inaccessible information about their biological families. PGT allows adoptees to uncover information about their genetic identities, thus helping them to fill the void of an incomplete family history. Both adoptees and nonadoptees reported improving their diet and exercise habits after PGT. Concerns that adoptees might give too much weight to PGT results appear to be unfounded, with adopted PGT customers no more likely to act on their results than nonadoptees.

\section{SUPPLEMENTARY MATERIAL}

Supplementary material is linked to the online version of the paper at http://www.nature.com/gim

\section{ACKNOWLEDGMENTS}

This work was supported by a National Institutes of Health R01 award (R01-HG005092). At the time this work was completed, D.A.C. was supported by a Canadian Institutes of Health Research Doctoral Foreign Study Award. R.C.G. is also supported by $\mathrm{NIH}$ grants U01-HG006500, U19-HD077671, and R01-HG002213. The content is solely the responsibility of the authors and does not necessarily represent the official views of the National Center for Research Resources, the National Institutes of Health, or the Canadian Institutes of Health Research.

Current members of the PGen Study Group are Robert C. Green, Joel B. Krier, Margaret H. Helm, and Lisa S. Lehmann of Harvard Medical School and Brigham and Women's Hospital; Deanna Alexis Carere and Peter Kraft of Harvard School of Public Health; J. Scott Roberts, Mack T. Ruffin IV, Lan Q. Le, and Jenny Ostergren of University of Michigan School of Public Health; Wendy R. Uhlmann and Mick P. Couper of University of Michigan; Joanna L. Mountain and Amy K. Kiefer of 23andMe; Glenn D. Braunstein of Pathway Genomics; Scott D. Crawford of Survey Sciences Group (SoundRocket); L. Adrienne Cupples, Clara A. Chen, and Catharine Wang of Boston University School of Public Health; Stacy W. Gray of Dana-Farber Cancer Institute; Barbara A. Koenig of University of California San Francisco; Kimberly Kaphingst of University of Utah; and Sarah Gollust of University of Minnesota.

\section{DISCLOSURE}

R.C.G.'s research is supported by grants from the $\mathrm{NIH}$, the DOD, and Illumina. R.C.G. has received compensation for advisory services or speaking from Invitae, Prudential, Illumina, AIA, Helix, and Roche. The other authors declare no conflict of interest.

\section{REFERENCES}

1. Guttmacher AE, Collins FS, Carmona RH. The family history-more important than ever. N Engl J Med 2004;351:2333-2336.

2. Rich EC, Burke $W$, Heaton $C J$, et al. Reconsidering the family history in primary care. J Gen Intern Med 2004;19:273-280.

3. Roberts JS, Ostergren J. Direct-to-Consumer Genetic Testing and Personal Genomics Services: A Review of Recent Empirical Studies. Curr Genet Med Rep 2013;1:182-200. 


\section{ORIGINAL RESEARCH ARTICLE}

4. 23andMeBlog Searching with 23andMe. 4 August. 2015. http://blog.23andme. com/23andme-customer-stories/searching-with-23andme/. Accessed 27 October 2015.

5. Su Y, Howard HC, Borry P. Users' motivations to purchase direct-to-consumer genome-wide testing: an exploratory study of personal stories. J Community Genet 2011;2:135-146.

6. Aiyar L, Shuman C, Hayeems R, et al. Risk estimates for complex disorders: comparing personal genome testing and family history. Genet Med 2014;16:231-237.

7. Heald B, Edelman E, Eng C. Prospective comparison of family medical history with personal genome screening for risk assessment of common cancers. Eur $J$ Hum Genet 2012;20:547-551.

8. US Food and Drug Administration. Warning letter to 23andMe.com. 22 November 2013. http://www.fda.gov/ICECI/EnforcementActions/ WarningLetters/2013/ucm376296. Accessed 27 October 2015.

9. Hill CM, Edwards M. Birth family health history: Adopters' perspectives on learning about their child's health inheritance. Adopt Foster 2009;33:45-53.

10. May T, Strong KA, Khoury MJ, Evans JP. Can targeted genetic testing offer useful health information to adoptees? Genet Med 2015;17:533-535.

11. Corder K. Counseling adult adoptees. Fam J 2012;20:448-452.

12. Bombard Y, Semaka A, Hayden MR. Adoption and the communication of genetic risk: experiences in Huntington disease. Clin Genet 2012;81:64-69.

13. Swarns RL. With DNA testing, suddenly they are family. The New York Times. 23 January 2012.

14. Carere DA, Couper MP, Crawford SD, et al.; PGen Study Group. Design, methods, and participant characteristics of the Impact of Personal Genomics (PGen) Study, a prospective cohort study of direct-to-consumer personal genomic testing customers. Genome Med 2014;6:96.

15. 23andMe. http://www.23andme.com. Accessed 27 October 2015

16. Pathway Genomics. http://www. pathway.com. Accessed 27 October 2015

17. Lehmann LS, Kaufman DJ, Sharp RR, et al. Navigating a research partnership between academia and industry to assess the impact of personalized genetic testing. Genet Med 2012;14:268-273.

18. Carere DA, Kraft P, Kaphingst KA, Roberts JS, Green RC. Consumers report lower confidence in their genetics knowledge following direct-to-consumer personal genomic testing. Genet Med 2016;18:65-72.

19. Ostergren JE, Gornick MC, Carere DA, et al.; PGen Study Group. How well do customers of direct-to-consumer personal genomic testing services comprehend genetic test results? findings from the impact of personal genomics study. Public Health Genomics 2015;18:216-224.
20. Meisel SF, Carere DA, Wardle J, et al.; PGen Study Group. Explaining, not just predicting, drives interest in personal genomics. Genome Med 2015;7:74.

21. Bowling A. Just one question: if one question works, why ask several? $J$ Epidemiol Community Health 2005;59:342-345.

22. National Center for Health Statistics. Adult physical activity questions on the national health interview survey: 1975-2012. 2012. http://www.cdc.gov/nchs/ data/nhis/physicalactivity/pa_questions.pdf. Accessed 27 October 2015.

23. Spitzer RL, Kroenke K, Williams JB, Löwe B. A brief measure for assessing generalized anxiety disorder: the GAD-7. Arch Intern Med 2006;166: 1092-1097.

24. Kroenke K, Spitzer RL, Williams JB. The Patient Health Questionnaire-2: validity of a two-item depression screener. Med Care 2003;41: 1284-1292.

25. Rumpf HJ, Meyer C, Hapke U, John U. Screening for mental health: validity of the MHI-5 using DSM-IV Axis I psychiatric disorders as gold standard. Psychiatry Res 2001:105:243-253.

26. Brehaut JC, O'Connor AM, Wood TJ, et al. Validation of a decision regret scale. Med Decis Making 2003:23:281-292.

27. Ryan GW, Bernard HR. Techniques to identify themes. Field Methods 2003:15:85-109.

28. Sobol MP, Cardiff J. A sociopsychological investigation of adult adoptees' search for birth parents. Fam Relat 1983:32:477-483.

29. McGowan ML, Fishman JR, Lambrix MA. Personal genomics and individual identities: motivations and moral imperatives of early users. New Genet Soc 2010;29:261-290

30. Crouch J, Yu J-H, Shankar A, Tabor H. "We don't know her history, her background": adoptive parents' perspectives on whole genome sequencing results. J Genet Counse/ 2014:1-11.

31. Hudson K, Javitt G, Burke W, Byers P; American Society of Human Genetics Social Issues Committee. ASHG statement on direct-to-consumer genetic testing in the United States. Obstet Gyneco/ 2007;110:1392-1395.

32. Caulfield T, McGuire AL. Direct-to-consumer genetic testing: perceptions, problems, and policy responses. Annu Rev Med 2012;63:23-33.

33. Kaufman DJ, Bollinger JM, Dvoskin RL, Scott JA. Risky business: risk perception and the use of medical services among customers of DTC personal genetic testing. J Genet Couns 2012;21:413-422.

34. Bloss CS, Schork NJ, Topol EJ. Effect of direct-to-consumer genomewide profiling to assess disease risk. N Eng/ J Med 2011;364:524-534.

35. Bloss CS, Topol EJ, Schork NJ. Association of direct-to-consumer genome-wide disease risk estimates and self-reported disease. Genet Epidemio/ 2012:36:66-70. 